\title{
Power Load Forecasting Model Based on Grey Neural Network Regression Combination
}

\author{
Ma Guozhen ${ }^{1}$, Pang Ning ${ }^{1}$, Zhang Zeya ${ }^{1}$, Wang Yongli ${ }^{2}$, Liu Chen ${ }^{2,}$, Yao Suhang ${ }^{2}$, Tao Siyi ${ }^{2}$ \\ ${ }^{1}$ Economic and Technological Research Institute of State Grid Hebei Electric Power Co., Ltd, No. 27, Fuqiang Street, Yuhua District, \\ Shijiazhuang ,Hebei, 050022, China \\ ${ }^{2}$ North China Electric Power University, Beijing, 102206, China
}

\begin{abstract}
Due to the limitations of a single power load forecasting model, the power load forecasting cannot be performed well. In order to obtain a greater closeness to predict results with actual data, this paper presents the power load forecasting model based on gray neural network combined return to Guangzhou, 2010 - 2019 on actual data for example, the results show that: As used herein, the combined model method has high accuracy and strong use value.
\end{abstract}

\section{Introduction}

Load forecasting is based on historical data such as power load, economy, society, weather, etc., to explore the influence of historical data changes of power load on future loads, and to seek the internal connection between power load and various related factors, so as to carry out future power loads. Scientific predictions. [1] At the same time, distributed energy and new energy storage devices are gradually being merged into the strong smart grid, and tidal load changes are increasing. Therefore, accurate short-term load forecasting becomes an important part of ensuring the safe and healthy operation of smart grids.effective precipitation. Moreover, there is a lack of overall planning at the business level, and business implementation is heavily dependent on frontend application systems, and effective collaboration between business modules across application systems cannot be achieved.

In order to improve the accuracy of short-term load forecasting, scholars at home and abroad have proposed many forecasting methods in the past decades [2], such as trend extrapolation, time series, regression analysis, etc. ${ }^{[3-5]}$ The shortcomings of these models are they only considers a growth trend, and cannot well reflect the seasonal trend of short-term load. Since the power load is a complex nonlinear system affected by many factors, and is especially sensitive to seasonal changes, it is not enough to consider only one growth trend. In response to these problems, this paper uses a combined forecasting model to verify the actual data of Guangzhou from 2010 to 2019 .

\section{Power load forecasting mechanism}

\subsection{Method improvement based on neural network}

The learning rule of BP network is to use gradient descent algorithm to optimize and adjust the parameters of the network according to the error gradient descent method, so that the error between the actual output value and the expected output value of the network can meet the accuracy requirements.[6] Considering the shortcomings of BP neural network, such as slow convergence speed and easy to fall into local optimum, Levenberg Marquardt numerical optimization algorithm (LM algorithm) is introduced to optimize the parameter adjustment process of BP neural network. LM algorithm combines the advantages of gradient descent algorithm and Gauss Newton method to accelerate the convergence speed and improve the accuracy of neural network, which effectively makes up for the shortcomings of traditional BP neural network.[7-8] Let the neural network have $\mathrm{m}$ input nodes in the input layer, $\mathrm{Q}$ neurons in the hidden layer and 1 output nodes in the output layer. The specific steps are as follows. The objective function of minimizing the daily operation cost of smart energy system is as follows:

1) Initialization: randomly initializes the weights of BP neural network and the threshold value of neurons.

2) Input training sample: the training sample set is $X=\left[X_{1}, X_{2}, \ldots, X_{n}\right]$, and any training sample is $X_{P}$ a m-dimensional column vector, that ${ }_{\text {is }} X_{P}=\left[x_{p 1}, x_{p 2}, \ldots, x_{p m}\right]^{T}$, the expected output ${ }_{\text {is }} T_{P}=\left[t_{p 1}, t_{p 2}, \ldots, t_{p l}\right]^{T}(P=1,2, \ldots, \mathrm{n})$

\footnotetext{
$\overline{{ }^{*} \text { Corresponding author: NCEPUliuchen } @ 163 . c o m}$
} 
3) Feedforward calculation: suppose a sample in the sample set is used to train the BP neural network. Firstly, the input and output values of each neuron in the hidden layer and output layer are calculated according to formula (1) to formula (4).

$$
\begin{aligned}
& \operatorname{net}_{i}^{p}=\sum_{j=1}^{M} \boldsymbol{w}_{i j} \boldsymbol{x}_{p j}-\boldsymbol{\theta}_{i} \\
& \boldsymbol{O}_{i}^{p}=g\left(\text { net }_{i}^{p}\right) \\
& \text { net }_{k}^{p}=\sum_{i=1}^{q} \boldsymbol{W}_{k i} \boldsymbol{O}_{i}^{p}-\boldsymbol{\theta}_{k} \\
& \boldsymbol{O}_{k}^{p}=g\left(\text { net }_{k}^{p}\right)
\end{aligned}
$$

4) Parameter adjustment: calculate the quadratic error function value under the action of samples according to formula (5). Using Levenberg Marquardt algorithm, the weights and thresholds of BP neural network are adjusted according to formula (6).

$$
\begin{gathered}
E_{P}=\frac{1}{2} \sum_{k=1}^{L}\left(t_{p k}-o_{k}^{p}\right)^{2}=\frac{1}{2} \sum_{k=1}^{L} e_{k}^{2} \\
\lambda_{k+1=} \lambda_{k}-\left[J\left(\lambda_{k}\right)^{T} J\left(\lambda_{k}\right)+\mu I\right]^{-1} J\left(\lambda_{k}\right)^{T} e\left(\lambda_{k}\right)
\end{gathered}
$$

5) Return to step 3). If the error meets the accuracy requirements, the learning is finished; otherwise, the next iteration is started.

\subsection{Grey load forecasting technology}

The modeling mechanism of grey model is one of the main research contents of grey system theory. The essence of grey modeling is to establish differential equation model based on a small amount of irregular historical data, so as to predict the development law of things. The specific process is as follows: firstly, in order to weaken the randomness of the data, the original data is accumulated to generate; secondly, the first-order differential equation model is constructed for the accumulated generated sequence; finally, the relevant parameters in the first-order differential equation model are estimated by using the least square method, and then the specific calculation formula of GM $(1,1)$ prediction model is obtained.

The original data sequence is

$$
X^{A}=\left[X^{A}(1), X^{A}(2), \ldots, X^{A}(n)\right]
$$

If it is satisfied with $X^{A}(\mathrm{~K}) \geq 0$, it is generated by first order accumulation

$$
X^{B}=\left[X^{B}(1), X^{B}(2), \ldots, X^{B}(n)\right]
$$

$$
\text { If it is satisfied with } X^{B}(\mathrm{k})=\sum_{i=1}^{k} x^{A}(\mathrm{i})
$$
order linear differential equation is constructed as follows:

$$
\frac{d_{x}{ }^{A}}{d_{t}}+a x^{A}=u
$$

The least square method is used to solve the parameters a, $\mathrm{U}$

$$
\left[\begin{array}{c}
\hat{a} \\
\hat{u}
\end{array}\right]=\left(B^{T} B\right)^{-1} B^{T} Y_{n}
$$

By substituting $\mathrm{a}^{\wedge}$ and $\mathrm{u}^{\wedge}$ back to the original differential equation, we can get

$$
x^{a}(\mathrm{k}+1)=\left[x^{a}(1)-\frac{\hat{u}}{\hat{a}}\right] e^{-\hat{a} k}+\frac{\hat{u}}{\hat{a}}
$$

The grey prediction model obtained by reduction is as follows:

$$
\hat{x}^{a}(\mathrm{k}+1)=\left(1-e^{\hat{a}}\right)\left[x^{a}(1)-\frac{\hat{u}}{\hat{a}}\right] e^{-\hat{a} k}
$$

The advantage of this model is that it does not need a large sample size and does not need to consider whether the data obey normal distribution. Through the accumulation technology, the data form an exponential law, thus establishing a unified differential equation. After obtaining the fitting curve, the future development value of the object is predicted. The grey prediction can predict the nonlinear system well.

In addition, in order to improve the prediction accuracy of the grey model, the original sequence of numbers is transformed before the accumulation generation, and the original sequence is transformed into the sequence with stable increase in exponential form as far as possible.[9] Suppose the original sequence is $X=\left\{x_{1}, x_{2}, \ldots, x_{n}\right\}$, and the original sequence is modified by adding policy factors. The expression of the transformed sequence

$$
\begin{gathered}
X_{z}=\left\{x_{1 z}, x_{2 z}, \ldots, x_{n z}\right\}, x_{i z} \text { shown in equation (13). } \\
x_{i z}=\frac{1}{n-i+1}\left(x_{i}+x_{i+1}+\ldots+x_{n}\right)
\end{gathered}
$$

\subsection{Multiple linear regression analysis}

Multiple linear regression prediction method is a popular and intuitive method among many prediction methods, and the key of using multiple linear regression prediction method to predict is to determine the multiple linear regression equation. The general form of multiple linear regression model is shown in equation (14).

$Y_{t}=b_{0}+b_{1} x_{1}+b_{2} x_{2}+\ldots+b_{m} x_{m}+\varepsilon$

Where $\mathrm{y}$ is the actual value matrix of dependent variable, $\mathrm{X}$ is the actual value matrix of independent variable, and $B$ is the undetermined coefficient matrix, and the formula is as follows: 


$$
X=\left[\begin{array}{ccccc}
1 & x_{11} & x_{12} & \cdots & x_{1 m} \\
1 & x_{21} & x_{22} & \cdots & x_{2 m} \\
1 & x_{31} & x_{32} & \cdots & x_{3 m} \\
\vdots & \vdots & \vdots & \cdots & \\
1 & x_{n 1} & x_{n 2} & \cdots & x_{n m}
\end{array}\right]
$$

Based on different prediction theory and technology, different prediction accuracy results will be obtained. Variance covariance method is used to assign more weight to the prediction value with higher accuracy, and the weighted average value is taken as the final prediction value to improve the prediction result. Taking the three prediction methods selected in this paper as examples, the calculation expression of weight is derived. $f_{\text {Let }}, f_{2}, f_{3}$ are the predicted values obtained by the three prediction methods respectively, and $\mathcal{W}_{1}$ 、

$w_{2} \quad$, $w_{3}$ are the weight coefficients $w_{1}+w_{2}+w_{3}=1$, and $f_{c}$ are the weighted average values. The prediction errors corresponding to $f_{1}, f_{2}, f_{3}, f_{c}$ are represented by $e_{1}, e_{2}, e_{3}, e_{c}$,the variance of prediction errors is represented by $D_{\left(e_{1}\right)}, D_{\left(e_{2}\right)}$ 、 $D_{\left(e_{3}\right)}, D_{\left(e_{c}\right)}$

Considering that the errors of different prediction methods are independent, the covariance between error variables is taken as 0 . The specific expression is shown in equations (16) to (18).

$$
\begin{aligned}
f_{c} & =w_{1} f_{1}+w_{2} f_{2}+w_{3} f_{3} \\
e_{c} & =w_{1} e_{1}+w_{2} e_{2}+w_{3} e_{3} \\
D_{\left(e_{c}\right)} & =w_{1}^{2} D_{\left(e_{1}\right)}+w_{2}^{2} D_{\left(e_{2}\right)}+w_{3}^{2} D_{\left(e_{3}\right)}
\end{aligned}
$$

Under the constraint conditions of, the Lagrange multiplier is introduced to find the minimum value of (I $=1,2,3)$, and the weight expression shown in equations (19) to (21) can be obtained.

$$
\begin{aligned}
& W_{1}=\frac{D_{\left(e_{2}\right)} D_{\left(e_{3}\right)}}{D_{\left(\varphi^{2}\right)} D_{\left(e_{2}\right)}+D_{\left(e_{1}\right)} D_{\left(e_{3}\right)}+D_{(e 2)} D_{\left(e_{3}\right)}} \\
& W_{2}=\frac{D_{(e) 1} D_{\left(e_{3}\right)}}{D_{\left(e^{2}\right)} D_{\left(e_{2}\right)}+D_{\left(e^{2}\right)} D_{\left(e_{3}\right)}+D_{(e 2)} D_{\left(e_{3}\right)}} \\
& W_{3}=\frac{D_{(e l)} D_{(e 2)}}{D_{\left(e^{2}\right)} D_{\left(e_{2}\right)}+D_{\left(e^{2}\right)} D_{\left(e_{3}\right)}+D_{(e 2)} D_{\left(e_{3}\right)}}
\end{aligned}
$$

\section{Case}

\subsection{Basic data}


$16411 \mathrm{mw}, 16913 \mathrm{mw}$ and $17334 \mathrm{mw}$ respectively. It can be seen from Table 1 that the comparison and analysis of single prediction methods are as follows: the accuracy of prediction values in 2010, 2012, 2015 and 2016 given by neural network prediction method is relatively high, and the accuracy of prediction values in 2010, 2013 and 2014 is relatively low. The accuracy of prediction value in 2011 and 2013 given by grey prediction method is relatively high, while that in 2014 and 2016 is relatively low; the accuracy of prediction value in 2010 and 2014 given by multiple linear regression prediction method is relatively high, and the accuracy of prediction value in 2015 is relatively low. It can be seen that the single prediction method may have a relatively high prediction accuracy in one year, but relatively low prediction accuracy in other years. The combined forecasting method proposed in this paper fully combines the advantages of the three prediction methods, effectively overcomes the shortcomings of strong randomness of single prediction method, and the accuracy and stability of prediction results are relatively high.

\section{Conclusion}

Considering the limitation of single forecasting method, this paper proposes an annual maximum load forecasting method based on grey neural network regression combination model. This method uses variance covariance method to optimize the weighted combination of BP neural network prediction method, gray prediction method and multiple linear regression method. It fully combines the advantages of neural network nonlinear mapping, gray prediction method dealing with small sample problems with incomplete information and linear mapping advantage of multiple linear regression method, which can improve the prediction effect and improve the prediction stability. The combined forecasting method proposed in this paper is verified by the actual data of Guangzhou City in historical years. The prediction results show that the proposed method has high prediction accuracy and good stability, which provides reliable technical support for the safe and economic operation and mode arrangement of power grid.

\section{Acknowledgments}

This article is funded by the Science and Technology Research Project of the Economic and Technical Research Institute of State Grid Hebei Electric Power Co., Ltd.: Research on seasonal power grid load characteristics and development trends based on data mining (SGHEJY00GHJS1900055).

\section{References}

1. Kang C.Q., Xia Q., Liu M. (2017) Power system load forecasting. M. Beijing: China Electric Power Press.
2. Kuster C., Rezgui Y., Mourshed M. (2017) Electrical load forecasting models: a critical systematic review. J. Sustainable Cities \& Society, 35 .

3. Huang J.B. (2019) Analysis of load development characteristics of urban complex based on trend extrapolation method. J. Rural Electrification, (07): $39-42$.

4. Zhao S., Cheng D., Chen Y., Jia Y. (2020) A time series based material forecasting method for distribution network. J. Zhejiang Electric Power, 39 (04): 52-56.

5. Wu F.H., Yang J.W. (2004) Several load forecasting methods and their comparison. J. Guangdong Electric Power, (01): 17-21.

6. GEETA P., Bhim S., BIJAYA K.P. (2016) Backpropagation algo-rithm-based controller for autonomous Wind-DG microgrid. J. IEEE Transactions on Industry Applications, 52 (5): 44084415.

7. FUXG, LISH, MICHAEL F.,etc. (2015) Training recurrent neu-ral net works with the levenbergmarquardt algorithm for optimal control of a gridconnected converter. J. IEEE Transactions on Neural Net Works and Learning Systems, 26 (9): 1900-1912.

8. Hou Y.1., Li T. (2008) BP neural network target recognition method based on LM optimization algorithm. J. Journal of Detection and Control, 30(1): 53-57. 